\title{
A Convex Max-Flow Segmentation of LV using Subject-Specific Distributions on Cardiac MRI
}

\author{
Mohammad Saleh Nambakhsh ${ }^{1,3}$, Jing Yuan ${ }^{4}$, Ismail Ben Ayed ${ }^{2}$, Kumaradevan \\ Punithakumar $^{2}$,Aashish Goela ${ }^{5,6}$, Ali Islam ${ }^{5,6}$, Terry Peters ${ }^{1,3}$, Shuo Li ${ }^{1,2}$ \\ 1 Biomedical Engineering Program, University of Western Ontario, London, Canada. \\ ${ }^{2}$ GE Healthcare, London, ON, Canada. \\ ${ }^{3}$ Imaging Research Laboratories, Robarts Research Institute, London, ON, Canada. \\ ${ }^{4}$ Computer Science department, University of Western Ontario, London, Canada. \\ 5 Department of Medical Imaging, University of Western Ontario, London, Canada. \\ ${ }^{6}$ St. Joseph Health Care, London, ON, Canada.
}

\begin{abstract}
This work studies the convex relaxation approach to the left ventricle (LV) segmentation which gives rise to a challenging multi-region seperation with the geometrical constraint. For each region, we consider the global Bhattacharyya metric prior to evaluate a gray-scale and a radial distance distribution matching. In this regard, the studied problem amounts to finding three regions that most closely match their respective input distribution model. It was previously addressed by curve evolution, which leads to sub-optimal and computationally intensive algorithms, or by graph cuts, which result in heavy metrication errors (grid bias). The proposed convex relaxation approach solves the LV segmentation through a sequence of convex sub-problems. Each sub-problem leads to a novel bound of the Bhattacharyya measure and yields the convex formulation which paves the way to build up the efficient and reliable solver. In this respect, we propose a novel flow configuration that accounts for labelingfunction variations, in comparison to the existing flow-maximization configurations. We show it leads to a new convex max-flow formulation which is dual to the obtained convex relaxed sub-problem and does give the exact and global optimums to the original non-convex sub-problem. In addition, we present such flow perspective gives a new and simple way to encode the geometrical constraint of optimal regions. A comprehensive experimental evaluation on sufficient patient subjects demonstrates that our approach yields improvements in optimality and accuracy over related recent methods.
\end{abstract}

\section{Introduction}

Left ventricle $(\mathrm{LV})$ segmentation of $2 \mathrm{D}$ cardiac magnetic resonance (CMR) is one of the fundamental steps to diagnose coronary heart disease charactrized by heart wall motion abnormalities, ventriculare enlargement, aneurysms, scars ( it occurs only on the myocardioum region), strain, EF and etc.

Manual segmentation of CMR images is extremely tedious and time consuming ${ }^{7}$. As such, automatic or semi-automatic algorithms are highly desired.

\footnotetext{
${ }^{7}$ Almost over 200 2D images per subject
} 
Despite so many studies in the literature allocated to the task, challenges inherent to CMR images such as including papillary muscles in blood cavity within endocardial region, and presence of noise and artifacts prevented the algorithms to sufficiently address the problem for routine clinical use [1].

The problem of myocardial segmentation is commonly formulated as an optimization of a cost functional of the image features, which typically are solved using active contours or discrete graph-cut methods. Active contours have been theprevalent choice in medical image analysis as they are able to introduce a wide range of photometric and geometric constraints [2-5]. Generally, these constraints use pixel-wise penalties learned from image models in the training set that suffer from unability to distinguish between connected cardiac regions with almost the same intensity profile [2]. Also, such choice suffers from local optima, slow convergence, high sensitivities to initialization, and numerical complexity as well.

Training-based algorithms have several drawbacks including the difficulty in capturing the inter- and intra-subject variations and pathological charactristics. They further need a large amount of training dataset[2]. The recent active curve studies in $[6,2,3]$ advanced it by building subject-specific models of a manually segmented frame in the current cardiac sequence.

In the discrete setting, although graph cut guarantees convergence to a global optima in nearly real time [7], it is unable to support global functionals that arise in our problem. Nevertheless, the recent works [8-10] use relaxations via bounds or approximations of the energy functional. Although these works led to substantial improvements over active contours in terms of speed, optimality, and accuracy, they still suffer from metrication errors [11].

Alternatively, continuous convex relaxation approaches share the advantages of both active curves and graph cuts, which recently have attracted a significant research attention in image segmentation problems. Some current studies based on convex optimization have shown its potential in solving the classical piecewise constant Mumford-Shah segmentation model [12], and its multiphase (multiregional) variant [13].

In this study, segmentation of the LV endo- and epi-cardial boundaries in a CMR sequence is formulated in an iterative convex max-flow relaxation approach with two original discrete cost functions. We solve a sequence of sub-problems which results in an algorithm robust to initial conditions. Unlike active contours, it also does not require a large number of iterative updates of the labeling and the time-consuming kernel density estimates (KDEs). The proposed formulation avoids a complex training process and the need for tremendous amount of training data. It leads to a segmentation faster than level-set methods and improvements in optimity and accuracy of the results. We further prove that the novel convex max-flow formulation obtains accurate and global solutions to the original, nonconvex sub-problems. Moreover, the proposed method is shapeinvariant and handles geometric variations of the LV intrinsically while it readily includes the papillary muscles into the endocardial or cavity region. 


\section{Convex Max-Flow Based Bhattacharyya Distribution Matching}

In this work, we make the multi-region segmentation of LV based on the principle of Bhattacharyya distribution matching. More specifically, our task is to find the optimal regions of Blood Cavity $\Omega_{c}$ and Myocardium $\Omega_{m}$, which best match the given distributions $\mathcal{M}_{c}$ and $\mathcal{M}_{m}$ based on the Bhattacharyya measure.

\subsection{Bhattacharyya Distribution Matching}

Let $I: \Omega \subset \mathbb{R}^{2} \rightarrow \mathcal{Z} \subset \mathbb{R}^{n}$ be an image function which maps domain $\Omega$ to the space $\mathcal{Z}$ of a photometric variable such as a color vector. Our studies in this work consist of the sub-problem to find the region $\mathcal{R} \subset \Omega$ whose distribution most closely matches the given reference distribution $\mathcal{M}$ by Bhattacharyya measure:

$$
\mathcal{B}(\mathcal{P}, \mathcal{M})=\sum_{z \in \mathcal{Z}} \sqrt{\mathcal{P}(z) \mathcal{M}(z)}
$$

where $\mathcal{P}(\mathrm{z})$ gives the nonparametric estimate of the distribution within $\mathcal{R}$ :

$$
\mathcal{P}(z)=\frac{\int_{\mathcal{R}} \mathcal{K}_{z}(x) d x}{|\mathcal{R}|}, \quad \forall z \in \mathcal{Z}
$$

with $|\mathcal{R}|$ the area of region $\mathcal{R}$, i.e. $|\mathcal{R}|=\int_{\mathcal{R}} d x ; \mathcal{K}_{z}(\cdot)$ is a kernel function, typically Gaussian:

$$
\mathcal{K}_{z}(x)=\frac{1}{\left(2 \pi \sigma^{2}\right)^{(n / 2)}} \exp \left(-\frac{\|z-I(x)\|^{2}}{2 \sigma^{2}}\right) .
$$

Studies such as [2] showed the Bhattacharyya distribution matching outperforms other methods in terms of robustness and accuracy.

In this study, our object is to find the optimal regions of Blood Cavity $\Omega_{c}$ and Myocardium $\Omega_{m}$, which match the given distributions $\mathcal{M}_{c}$ and $\mathcal{M}_{m}$ respectively, subject to the minimum total perimeter of the two regions $\Omega_{c}$ and $\Omega_{m}$, i.e. tight region bondaries are prefered. Beside this, we naturally adopt the geometrical constraint $\mathcal{R}_{c} \subset \mathcal{R}_{m}$ in our segmentation approach.

The problem therefore reduces to the following optimization problem:

$$
\min _{\mathcal{R}_{c}, \mathcal{R}_{m}}-\mathcal{D}_{c}\left(\mathcal{R}_{c}\right)-\mathcal{D}_{m}\left(\mathcal{R}_{m}\right)+\lambda\left(\left|\partial \mathcal{R}_{c}\right|+\left|\partial \mathcal{R}_{m}\right|\right)
$$

subject to the geometrical prior

$$
\mathcal{R}_{c} \subset \mathcal{R}_{m}
$$

where $\left|\partial \mathcal{R}_{c, m}\right|$ measures the perimeter of $\mathcal{R}_{c}$ and $\mathcal{R}_{m}$ respectively and first and second terms in (4) are defined in the following:

$$
\mathcal{D}_{c}\left(\mathcal{R}_{c}\right)=\alpha_{c} \mathcal{B}\left(\mathcal{P}_{c}^{I}, \mathcal{M}_{c}^{I}\right)+\left(1-\alpha_{c}\right) \mathcal{B}\left(\mathcal{P}_{c}^{D}, \mathcal{M}_{c}^{D}\right)
$$




$$
\mathcal{D}_{m}\left(\mathcal{R}_{m}\right)=\alpha_{m} \mathcal{B}\left(\mathcal{P}_{m}^{I}, \mathcal{M}_{m}^{I}\right)+\left(1-\alpha_{m}\right) \mathcal{B}\left(\mathcal{P}_{m}^{D}, \mathcal{M}_{m}^{D}\right)
$$

where first term in both (5) and (6) is the Bhattacharyya of intensity distribution and the second term is the Bhattacharyya of radial distance distribution (distance from centroid of the regions). Note that in (6) only the myocardial strip is included in the intesnsity distribution and the cavity region is excluded from the intensity distributions. $\alpha_{c}, \alpha_{m}$ are weights of the cavity and the myocardial regions which weights the intensity and the radial distance constraints.

\subsection{Alternating Optimization Approach}

For (4), we propose an effective alternating-optimization approach which splits (4) into two subproblems and tackles them sequentially at each iteration, see Alg. 1.

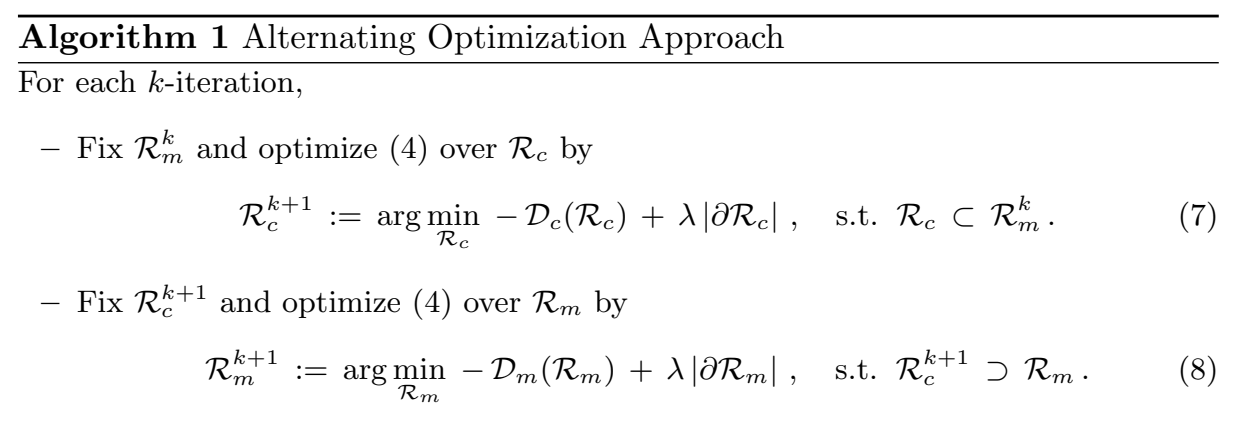

In view of the two subproblems (7) and (8), they essentially share the same type of optimization formulation which solves

$$
\min _{\mathcal{R}}-\mathcal{D}(\mathcal{R})+\lambda|\partial \mathcal{R}|
$$

subject to the respective geometrical constraint in (7) and (8).

In the following, we therefore focus on the optimization problem (9), which corresponds to two subproblems (7) and (8) with incorporated geometricalpriors. We further show it can be optimized globally and effectively by a novel iterative convex max-flow approach. It easily adapts the geometrical constraint by using the sufficiently large flow capacities (data cost) as standard max-flow scheme [14]. To this end, we only consider the optimization of (9) without any geometrical constraint which is assumed to be presented in flow settings.

\subsection{Global Minimization of Energy Upper Bound}

Let $u: \Omega \rightarrow\{0,1\}$ be the labeling function of $\mathcal{R} \subset \Omega$, i.e. $u(x)=1$ when $x \in \mathcal{R}$ and $u(x)=0$ otherwise. The distribution in (2) can be rewritten, in terms 
of $u(x)$, as:

$$
\mathcal{P}(z)=\frac{\int_{\Omega} \mathcal{K}_{z}(x) u(x) d x}{\int_{\Omega} u(x) d x}, \quad \forall z \in \mathcal{Z}
$$

then the Bhattacharyya measure of (1) is:

$$
\mathcal{B}(u, \mathcal{M})=\sum_{z \in \mathcal{Z}}\left(\frac{\int_{\Omega} \mathcal{T}_{M, z}(x) u(x) d x}{\int_{\Omega} u(x) d x}\right)^{1 / 2}
$$

where $\mathcal{T}_{M, z}(x)=\mathcal{K}_{z}(x) \mathcal{M}(z)$.

The perimeter of a region $\mathcal{R}$ can be evaluated by the weighted boundary of the region or indicator function $u(x) \in\{0,1\}[15,16]$ :

$$
|\partial \mathcal{R}|=\int_{\Omega} C(x)|\nabla u(x)| d x, \quad C(x) \geq 0 .
$$

In this paper, we focus on the case where $C(x)=\lambda$ is a positive constant. The results can be easily extended to the more general $C(x)$.

In view of (11) and (12), (9) can be reformulated by:

$$
\min _{u(x) \in\{0,1\}}\left\{E(u):=-\sum_{z \in \mathcal{Z}}\left(\frac{\int_{\Omega} \mathcal{T}_{M, z}(x) u(x) d x}{\int_{\Omega} u(x) d x}\right)^{1 / 2}+\int_{\Omega} C(x)|\nabla u(x)| d x\right\} .
$$

Direct computation of (13) is challenging, both theoretically and numerically, due to the constraint of a pointwise binary variable and the nonlinearity of its energy function ${ }^{8}$. To this end, we propose a novel iterative convex relaxation solution to (13), which globally minimizes a sequence of upper bounds of $E(u)$, denoted $E^{i+1}\left(u ; u^{i}\right), i=1 \ldots$ :

$$
u^{i+1}=\arg \min _{u \in\{0,1\}} E^{i+1}\left(u ; u^{i}\right), \quad i=1 \ldots
$$

where

$$
E(u) \leq E^{i+1}\left(u ; u^{i}\right), \quad i=1 \ldots
$$

and

$$
\left\|E(u)-E^{i+1}\left(u ; u^{i}\right)\right\| \rightarrow 0 \text {, when }\left\|u-u^{i}\right\| \rightarrow 0
$$

One can easily show that the sequence of solutions $u^{i}$ to the problems in (14) under constraints (15) and (16) yields a monotonically decreasing sequence of cost functions $E$ and, therefore, a minimum of $E$ at convergence, which can be summarized as the following proposions:

Proposition 1. Sequence $E\left(u^{i}\right)$ is monotonically decreasing, i.e. $E\left(u^{i+1}\right) \leq$ $E\left(u^{i}\right)$.

Proposition 2. Sequence $E\left(u^{i}\right)$ is convergent.

Due to limited space Proofs of Prop. 1 and Prop. 2 is presented on request.

\footnotetext{
${ }^{8}$ unlike the classical continuous min-cut problem in [12,17], (13) is nonconvex even when $u \in\{0,1\}$ is relaxed by $u \in[0,1]$.
} 


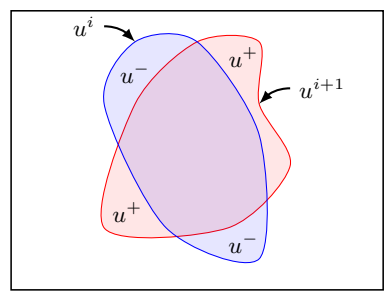

(a)

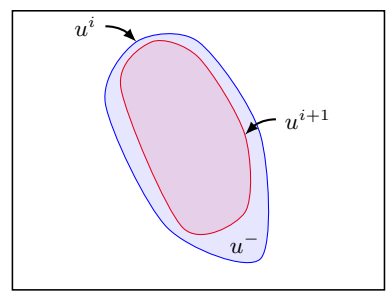

(b)

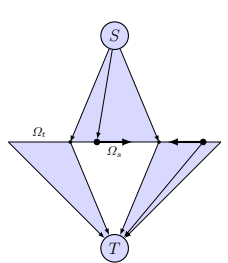

(c)

Fig. 1. Iterative bound optimization: (a) Illustrates the proposed bound; (b) Illustrates the bound in [9]. $u^{i}(x)$ denotes the labeling at iteration $i$; (c)The proposed spatially continuous max-flow configuration

Estimation of Energy Upper Bounds In view of Prop. 1 and Prop. 2, we derive estimation of the energy upper bound $E^{i+1}\left(u ; u^{i}\right)$, which is convex with respect to labeling function $u(x)$. We will further show that $E^{i+1}\left(u ; u^{i}\right)$ can be minimized globally and exactly over $u(x) \in\{0,1\}$ in the following part.

Let $u^{i}(x) \in\{0,1\}$ denote the binary labeling function, obtained at the previous $i$-th step, as the starting point of the $(i+1)$-th iteration. At this stage, we are seeking an optimal labeling function $u(x) \in\{0,1\}$ which minimizes the energy upper bound. Let us express the difference between two functions $u(x)$ and the given $u^{i}(x)$ as a function of two new variables $u^{+}(x) \in\{0,1\}$ and $u^{-}(x) \in\{0,1\}$ (refer to Fig. 1a):

1. $u^{+}(x)$ indicates the area where $u^{i}(x)$ is 0 and $u(x)$ becomes 1 , i.e. area increase:

$$
u^{+}(x):= \begin{cases}u(x), & \text { where } u^{i}(x)=0 \\ 0, & \text { otherwise }\end{cases}
$$

2. $u^{-}(x)$ indicates the area where $u^{i}(x)$ is 1 and $u(x)$ becomes 0 , i.e. area decrease:

$$
u^{-}(x):= \begin{cases}1-u(x), & \text { where } u^{i}(x)=1 \\ 0, & \text { otherwise }\end{cases}
$$

The following describes the upper bound we propose.

Proposition 3. Given a labeling $u^{i}(x) \in\{0,1\}$, for any labeling function $u(x) \in$ $\{0,1\}$, we have the following upper bound which depends on $u^{+}(x)$ and $u^{-}(x)$ :

$$
E(u) \leq-\mathcal{D}\left(u^{i}\right)+F^{i+1}\left(u ; u^{i}\right)
$$

where

$$
F^{i+1}\left(u ; u^{i}\right)=\int_{\Omega} C_{v}^{i} u^{-} d x+\int_{\Omega} C_{w}^{i} u^{+} d x+\int_{\Omega} C|\nabla u| d x
$$


and

$$
\begin{aligned}
& C_{v}^{i}(x)=\sum_{z \in \mathcal{Z}} \frac{\mathcal{D}_{z, i} \mathcal{T}_{M, z}(x)}{\int_{\Omega} \mathcal{T}_{M, z}(x) u^{i}(x) d x}, \quad C_{w}^{i}(x)=\sum_{z \in \mathcal{Z}} \frac{\mathcal{D}_{z, i}}{2 \int_{\Omega} u^{i}(x) d x}, \\
& \mathcal{D}_{z, i}=\left(\frac{\int_{\Omega} \mathcal{T}_{M, z}(x) u^{i}(x) d x}{\int_{\Omega} u^{i}(x) d x}\right)^{1 / 2} .
\end{aligned}
$$

Due to limited space a detailed proof of Prop. 3 is given on request.

Prop. 3 leads us to the following conclusions:

- Energy upper bound: In view of (19), we have the energy upper bound

$$
E^{i+1}\left(u ; u^{i}\right)=-\mathcal{D}\left(u^{i}\right)+F^{i+1}\left(u ; u^{i}\right)
$$

where $\mathcal{D}\left(u^{i}\right)$ is a constant corresponding to the given $u^{i}$.

- Continuous min-cut model: Hence we can minimize $E^{i+1}\left(u ; u^{i}\right)$ over $u(x) \in\{0,1\}$ by

$$
\min _{u(x) \in\{0,1\}} F^{i+1}\left(u ; u^{i}\right)
$$

where $F^{i+1}\left(u ; u^{i}\right)$ is given in (20). We call (23) the continuous min-cut model. It then proposes the following iterative minimization procedure:

1. Start with an arbitrary initial labeling $u^{1}(x)(i=1)$;

2. For the $i$-th outer iteration, set the flow configuration based on labeling function $u^{i}(x)$. Compute $C_{v}^{i}(x)$ and $C_{w}^{i}(x)$ by (21), and solve (23) to obtain $u^{i+1}(x) \in\{0,1\}$. In the following section, we propose a fast convex max-flow approach to computing (23) globally and exactly;

3 . Let $i=i+1$ and repeat the above two steps until convergence.

\section{Continuous Max-Flow Approach}

The new continuous min-cut model in (23) depends on labeling-function variations $\left(u^{+}\right.$and $\left.u^{-}\right)$, not on a single labeling function as with previous models [18, $12,19,17]$. In this section, we propose and solve the convex relaxation of $(23)$, called convex relaxed min-cut model, through its dual model, so-called convex max-flow model which is constructed upon a novel flow configuration (see Fig. 1c). We show the proposed convex relaxed min-cut model solves (23) exactly and globally by means of its convex max-flow model. The convex max-flow formulation naturally leads to a fast algorithm to $(23)[19,17]$.

\subsection{Convex Relaxed Min-Cut Model}

Given the current labeling function $u^{i}(x) \in\{0,1\}$, we define:

$$
\Omega=\Omega_{s} \cup \Omega_{t}, \quad \Omega_{s} \cap \Omega_{t}=\emptyset
$$

where $\Omega_{s}$ is indicated by $u^{i}=1$ and $\Omega_{t}$ by $u^{i}=0$. 
From the definitions of $u^{+}(17)$ and $u^{-}$(18), we reformulate (23) by:

$$
\min _{u(x) \in\{0,1\}} \int_{\Omega_{t}} C_{v}^{i}(1-u) d x+\int_{\Omega_{s}} C_{w}^{i} u d x+\int_{\Omega} C(x)|\nabla u| d x
$$

and propose its convex relaxation, i.e. convex relaxed min-cut model, by:

$$
\min _{u(x) \in[0,1]} \int_{\Omega_{t}} C_{v}^{i}(1-u) d x+\int_{\Omega_{s}} C_{w}^{i} u d x+\int_{\Omega} C(x)|\nabla u| d x .
$$

\subsection{Convex Max-Flow Model}

We propose the novel configuration of flows (see Fig. 1c for a 1D example) which accounts for the previous partition defined by $\Omega_{s}$ and $\Omega_{t}$, which is in contrast the existing continuous flow setting [19,17]. Given $\Omega_{s}$ and $\Omega_{t}$, we define three types of flows: the source flow $p_{s}(x)$ directed from $s$ to $\forall x \in \Omega_{s}$, the sink flow $p_{t}(x)$ directed from $\forall x \in \Omega_{t}$ to $t$, and the spatial flow $p(x)$ given $\forall x \in \Omega$.

$p_{s}(x), p_{t}(x)$ and $p(x)$ are constrained by the following flow capacities:

$$
p_{s}(x) \leq C_{v}^{n}(x), \quad p_{t}(x) \leq C_{w}^{n}(x), \quad|p(x)| \leq C(x), \quad \text { a.e } x \in \Omega ;
$$

and flow conservation conditions:

$$
-p_{s}(x)+\operatorname{div} p(x)=0, p_{t}(x)+\operatorname{div} p(x)=0, \quad \text { a.e. } x \in \Omega_{t} .
$$

The continuous max-flow model can, therefore, be formulated by maximizing the total flow from the source $s$ :

$$
\max _{p_{s}, p_{t}, p} \int_{\Omega_{s}} p_{s} d x
$$

subject to the flow constraints (27) and (28).

By means of convex analysis, we can prove the following result:

Proposition 4. For the convex models (26) and (29), we have the equivalence:

$$
(29) \Longleftrightarrow(26) \text {. }
$$

Due to limited space, its proof is given on request.

In addition, we can further show that the binary-valued optimization problem in (25) can be solved globally and exactly by its convex relaxation (26). Clearly, (26) is convex. Let $u^{*}(x)$ be its global optimum. We define the $\gamma$-upper level set $u_{\gamma}^{*}(x) \forall x \in \Omega$, for any $\gamma \in[0,1]$ :

$$
u_{\gamma}^{*}(x)= \begin{cases}1, & \text { when } u^{*}(x)>\gamma \\ 0, & \text { when } u^{*}(x) \leq \gamma\end{cases}
$$

Therefore, we have

Proposition 5. When $u^{*}(x)$ gives one global optimum of (26), its thresholding $u_{\gamma}^{*}(x) \in\{0,1\}$ by $(30)$, for any $\gamma \in[0,1]$, solves the binary-valued optimization problem in (25) globally and exactly.

The proof of Prop. 5 follows the ideas of $[19,17]$ (proposed on the request). 


\subsection{Convex Max-Flow Based Algorithm}

We propose a fast algorithm based on the convex max-flow model (29). In this regard, we define the following augmented Lagrangian function:

$$
\begin{aligned}
L_{c}\left(p_{s}, p_{t}, p, u\right)= & \int_{\Omega_{s}} p_{s} d x+\int_{\Omega_{s}}\left(\operatorname{div} p-p_{s}\right) u d x+\int_{\Omega_{t}}\left(\operatorname{div} p+p_{t}\right) u d x \\
& -\frac{c}{2}\left\|\operatorname{div} p-p_{s}\right\|_{\Omega_{s}}^{2}-\frac{c}{2}\left\|\operatorname{div} p+p_{t}\right\|_{\Omega_{t}}^{2}
\end{aligned}
$$

where $c>0$. Finally, we derive the continuous max-flow algorithm (Alg. ) from the classical augmented Lagrangian method [20,21], whose convergence properties can be found in [22].

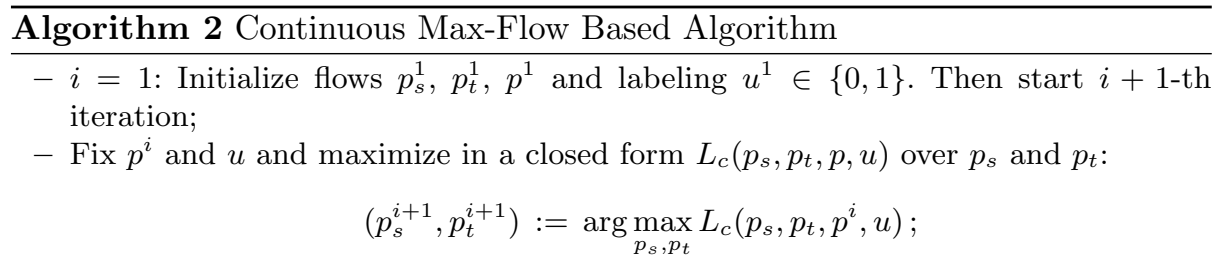

- Fix $p_{s}^{i+1}, p_{t}^{i+1}$ and $u$ and maximize iteratively $L_{c}\left(p_{s}, p_{t}, p, u\right)$ over $p$ via the simple projection-descent step:

$$
p^{i+1}:=\arg \max _{p} L_{c}\left(p_{s}^{i+1}, p_{t}^{i+1}, p, u\right) ;
$$

- Update labeling function $u$ by

$$
u(x)=\left\{\begin{array}{l}
u(x)+c\left(\operatorname{div} p^{i+1}(x)-p_{s}^{i+1}(x)\right), \forall x \in \Omega_{s} \\
u(x)+c\left(\operatorname{div} p^{i+1}(x)+p_{t}^{i+1}(x)\right), \forall x \in \Omega_{t}
\end{array} .\right.
$$

Repeat the above two steps until convergence.

\section{Experiments}

To evaluate the proposed method $(C M F M), 20$ subjects with 120 short axis cardiac cine $C M R I$ with pixel spacing of $0.7812 \times 0.7812$ and slice thickness of $10 \mathrm{~mm}$ including apical, mid-cavity, and basal slices were used. The manual segmentation of the first frame of each slice was chosen to learn the model distribusions. For evaluation purpose, automatic segmented cavity and myocardium were compared to the corresponding manually segmented regions. Also, for comparison the level-set segmentation $(L S M)$ in [2] and max-flow( graph-cut) method $(M F M)$ in [10] were run over the same datasets.

After tuning of the algorithm in terms of the different parameters, the following parameters were used for all the dataset identically. $\sigma=10^{-1}$ for the intensity distributions of the myocardium, $\sigma=10$ for the intensity distributions of the cavity, and $\sigma=2$ for the distance distributions in (3); $\alpha_{c}=0.95$ in (5) 
and $\alpha_{m}=0.6$ in (6). We also used $\lambda=0.3$ in (27) and $c=0.05$ in (31) for the convex max-flow optimizer.

\section{1 illustration of segmented images and quantitative evaluations}

Fig.2 shows the results of one subject over mid-cavity, apical, and basal slices. The yellow and red curves correspond to the endo- and epicardium boundaries, respectively. The third row shows the apical frames where it is challenging to segment the cavity as it is a small region with motion artifacts. Examples illustrate that the proposed method perfectly excludes the papillary muscles from the myocardium. The results also demonstrate that the proposed method handles wide variations of the cavity in terms of scale and shape.
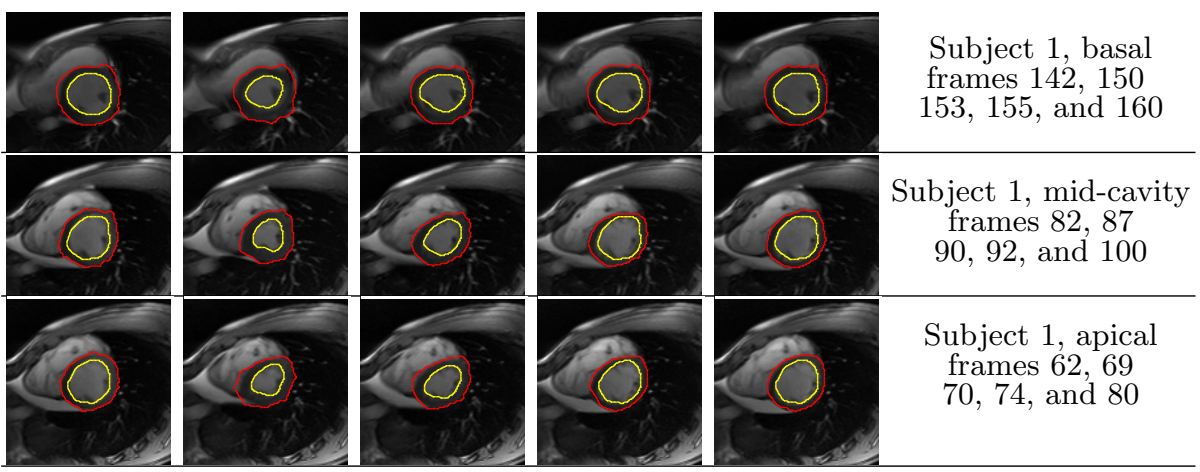

Fig. 2. Results for one subject at different slice levels: Basal (1st row); Mid-cavity(2nd row); Apical(3th row).

We further assessed the similarities between the ground truth and the segmentations via contour-based measure such as the Root Mean Squared Error $(R M S E)$, and region-based measure such as Dice Metric $(D M)$. Let $V_{a}$ and $V_{m}$ be automated and manually segmented volums. DM is given by $D M=2 \frac{V_{a} \cap V_{m}}{V_{a}+V_{a}}$. $D M$ measures the similarity between the automatically detected and groundtruth regions. Higher $D M$ shows better performance while it is always between 0 and 1. RMSE evaluates automatic epi- and endocardium boundaries by assigning 240 points along the boundary and computing the perpendicular distances between manual contour and them [10]. The lower RMSE shows the results are more close to the ground truth.

We examined quantitatively and qualitatively the reliability of the algorithm by evaluating the reliability function, i.e., the complementary cumulative distribution function (ccdf) of the obtained Dice metrics (Fig.3a and b).

Furthermore, one of the important quantitative clinical measures is LV volume which is the volume within the cavity. Fig.3c shows comparison of automated cavity volume and the manual one for 20 subjets over 20 phases. 
Cavity detection

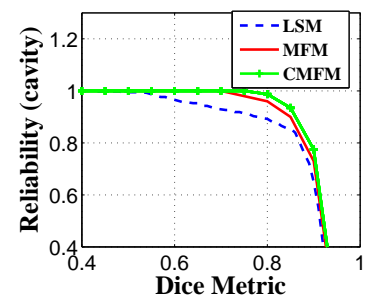

(a)

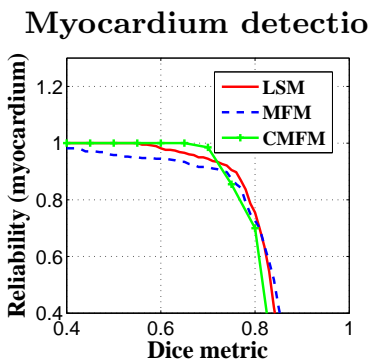

(b)

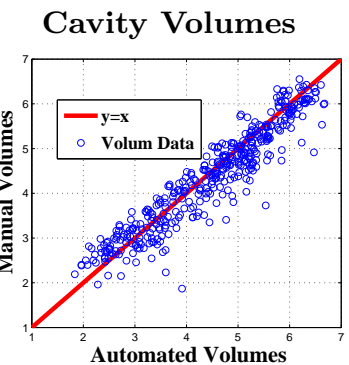

(c)

Fig. 3. Comparisons of (a) Cavity and (b)Myocardioum Dice Metric reliability $(\mathcal{R}(d)=$ $\operatorname{Pr}(D M>d) d$ : horizentalaxes $)$ for 2280 images acquired from 20 subjects by the proposed method (CMFM), MFM [10] and LSM [2] algorithms; (c) Comparisons of manual and automatic segmented volumes of the cavity for the same database.

Table 1 reports the $D M$ statistics, reliability of $D M$ higher than (0.80), average RMSE and average time is consumed to process a frame over 20 subjects for the proposed method (CMFM), MFM [10] and LSM [2].

\begin{tabular}{|c|c|c|c|c|c|c|c|}
\hline \multirow[t]{2}{*}{ Methods } & \multicolumn{2}{|c|}{ Dice Metric } & \multicolumn{2}{|c|}{ Reliability $(d=0.8)$} & \multicolumn{2}{|r|}{$R M S E$} & \multirow{2}{*}{$\begin{array}{l}\text { time per } \\
\text { frame (sec) }\end{array}$} \\
\hline & Cavity & Myocardium & Cavity & Myocardium & Cavity & Myocardium & \\
\hline CMFM & $0.92 \pm 0.07$ & $0.80 \pm 0.10$ & 1 & 0.70 & 1.35 & 2.05 & 1.79 \\
\hline MFM & $0.91 \pm 0.04$ & $0.81 \pm 0.06$ & 0.97 & 0.79 & 1.60 & 1.99 & 0.14 \\
\hline $\operatorname{LSM}[2]$ & $0.88 \pm 0.09$ & $0.81 \pm 0.10$ & 0.89 & 0.75 & 2.46 & 1.89 & 15.96 \\
\hline
\end{tabular}

Table 1. $D M$, Reliability of $D M$, the average $R M S E$ (in pixels), and average time load to process a frame over 20 subjects (2280 images) for the proposed method (CMFM), $M F M[10]$ and $L S M[2]$; Statistics of the $D M$ expressed as mean \pm standard deviation; Algorithms have been run over $2 \mathrm{GHZ} C P U$.

\section{Conclusion}

This study investigated fast detection of the $L V$ endo- and epicardial boundaries in a cardiac magnetic resonance $(M R)$ sequence. The solution is obtained by the proposed convex relaxation optimization of two original discrete cost functions constructed of a global geometric and a global photometric constraint based on the Bhattacharyya similarity. Quantitative evaluations demonstrated that the results correlate well with independent manual segmentations drawn by an expert. The proposed method outperformed a recent active contour method in [2], and graph-cut method in [10]. The proposed approach removes the need of a complex training, while handles intrinsically geometric variations of the $L V$ without biasing the solution towards a finite set of shapes. It also includes papillary muscles in the endocardial region.

\section{References}

1. Jolly, M.P.: Automatic recovery of the left ventricular blood pool in cardiac cine MR images. In: MICCAI 2008. Volume 1., New York, NY, USA (2008) 110-118 
2. Ben Ayed, I., Li, S., Ross, I.: Embedding overlap priors in variational left ventricle tracking. IEEE Trans. Med. Imaging 28(12) (2009) 1902-1913

3. Ben Ayed, I., Li, S., Ross, I., Islam, A.: Myocardium tracking via matching distributions. Int J of Comput. Assist Radiol. and Surg. 4(1) (2009) 37-44

4. Kaus, M.R., von Berg, J., Weese, J., Niessen, W., Pekar, V.: Automated segmentation of the left ventricle in cardiac MRI. Med. Image Anal. 8(3) (2004) 245-254

5. Liu, H., Chen, Y., Ho, H.P., Shi, P.: Geodesic active contours with adaptive neighboring influence. In: MICCAI 2005. Volume 2., CA, USA 741-748

6. Zhu, Y., Papademetris, X., Sinusas, A.J., Duncan, J.S.: Segmentation of the left ventricle from cardiac MR images using a subject-specific dynamical model. IEEE Trans. Med. Imaging 29(4) (2010) 669-687

7. Boykov, Y., Funka Lea, G.: Graph cuts and efficient N-D image segmentation. Int. J. Comput. Vision 70(2) (2006) 109-131

8. Rother, C., Minka, T., Blake, A., Kolmogorov, V.: Cosegmentation of image pairs by histogram matching - incorporating a global constraint into MRFs. In: CVPR 2006

9. Ben Ayed, I., Chen, H.M., Punithakumar, K., Ross, I., Li, S.: Graph cut segmentation with a global constraint: Recovering region distribution via a bound of the Bhattacharyya measure. In: CVPR 2010

10. Ben Ayed, I., Punithakumar, K., Li, S., Islam, A., Chong, J.: Left ventricle segmentation via graph cut distribution matching. In: MICCAI 2009. 901-909

11. Pock, T., Chambolle, A., Cremers, D., Bischof, H.: A convex relaxation approach for computing minimal partitions. In: CVPR 2009

12. Nikolova, M., Esedoglu, S., F., T.: Algorithms for finding global minimizers of image segmentation and denoising models. SIAM J. Appl. Math. 66(5) (2006) $1632-1648$

13. Lellmann, J., Kappes, J., Yuan, J., Becker, F., Schnörr, C.: Convex multi-class image labeling by simplex-constrained total variation. Tech report, HCI, IWR, Uni. Heidelberg (2008)

14. Boykov, Y., Kolmogorov, V.: An experimental comparison of min-cut/max-flow algorithms for energy minimization in vision. IEEE Trans. Pattern Anal. Mach. Intell. 26 (September 2004) 1124-1137

15. Chan, T., Shen, J.H.: Image Processing And Analysis: Variational, PDE, Wavelet, and Stochastic Methods. SIAM, Philadelphia, PA, USA (2005)

16. Giusti, E.: Minimal surfaces and functions of bounded variation. Australian National University, Canberra (1977)

17. Yuan, J., Bae, E., Tai, X.C., Boycov, Y.: A study on continuous max-flow and mincut approaches. Part I: Binary labeling. Tech report CAM-10-61, UCLA (2010)

18. Bresson, X., Esedoglu, S., Vandergheynst, P., Thiran, J., Osher, S.: Fast global minimization of the active contour/snake model. J. Math. Imaging Vis. 28(2) (2007) 151-167

19. Yuan, J., Bae, E., Tai, X.C.: A study on continuous max-flow and min-cut approaches. In: CVPR 2010

20. Rockafellar, R.T.: The multiplier method of Hestenes and Powell applied to convex programming. J. Optimiz. Theory App. 12 (1973) 555-562

21. Rockafellar, R.T.: Augmented Lagrangians and applications of the proximal point algorithm in convex programming. Math. Oper. Res. 1(2) (1976) 97-116

22. Bertsekas, D.P.: Nonlinear Programming. Athena Scientific (1999) 